\title{
Repair of a Traumatic Lumbar Hernia With Biosynthetic Mesh: A Novel Approach and Review of the Literature
}

\author{
Beth-Ann Shankera, Victoriya Sara Chernyavsky ${ }^{a}$, Kelly Johnson a, Joshua N. Honeymana, \\ Tomer Davidov ${ }^{\mathrm{b}}$
}

\begin{abstract}
Lumbar hernias are a rare type of abdominal hernia which occurs from a defect in the posterior-lateral abdominal wall. Lumbar hernias are commonly acquired through trauma, surgery, and infection. The most common etiology is a blunt abdominal trauma associated with crush injuries, or restrained victims of a motor vehicle collision. Different surgical techniques to repair these hernias have been described. We report on the use of a biosynthetic mesh as an adjunct to repairing a traumatic lumbar hernia, which has a higher likelihood of contamination or infection secondary to traumatic etiology. We also review the literature on this topic of lumbar hernia repair.
\end{abstract}

Keywords: Lumbar hernia; Biologic mesh repair

\section{Introduction}

Lumbar hernia is rare type of abdominal hernia which occurs from a defect in the posterolateral abdominal wall through which either intraperitoneal or extraperitoneal contents protrude. While the first descriptions of lumbar hernias date to

Manuscript accepted for publication April 18, 2012

${ }^{a}$ Division of General Surgery, University of Medicine and Dentistry of New Jersey, Robert Wood Johnson Medical School, Office of Surgical Education, MEB 596, P.O. Box 19, New Brunswick, NJ 08903-0019, USA

${ }^{\mathrm{b}}$ Corresponding author: Division of General Surgery, University of Medicine and Dentistry of New Jersey, Robert Wood Johnson Medical School, Office of Surgical Education, MEB 452, P.O. Box 19, New Brunswick, NJ 08903-0019, USA. Email: davidoto@umdnj.edu

doi: $10.4021 / \mathrm{jcs} 47 \mathrm{~W}$ the mid-1600s, current medical literature describes approximately 300 cases [1].

The two most common anatomical locations of lumbar hernias are the superior and inferior lumbar triangles, whose boundaries have been well described. The superior triangle, which was first described by Joseph Grynfeltt in 1866, is bordered superiorly by the inferior edge of the 12th rib and the serratus posterior inferior muscle, laterally by the internal oblique, and medially by the quadratus lumborum (Fig. 1). In 1783 Jean Louis Petit described the inferior lumbar region as bound by the latissimus dorsi, the free margin of the external oblique, and the superior edge of the iliac crest (Fig. 2 ). The floor of the triangle is composed of lumbodorsal fascia and the transversalis abdominus muscle aponeurosis [2].

Causes of lumbar hernia can be divided into primary hernias and secondary hernias. Primary lumbar hernias comprise $20 \%$ and include spontaneous hernias and the lumbocostovertebral syndrome, in which congential hernia is associated with rib and vertebral anomalies. Secondary, or acquired, lumbar hernias account for $80 \%$ and are associated with trauma, surgery, and infection [3]. The most common etiology for lumbar hernias is blunt abdominal trauma associated with crush injuries or restrained victims of motor vehicle collisions [4].

The points of anatomic weaknesses usually occur at the apices or the triangle floors. Herniation through either lumbar triangle may be associated with bowel incarceration or strangulation or herniation of other intraabdominal organs $[5,6]$. Blunt deceleration traumatic injury, coupled with abdominal restraints, results in sudden increase in intraabdominal pressure and transmission of opposing shearing forces. The herniation results at the maximum point of weakness, with concentrated force transmitted at the junction of the shoulder and lap belt, which is most commonly the apex of the inferior lumbar triangle [7].

The diagnosis of lumbar hernia is initially clinical, however the use of imaging to both diagnose and describe lumbar hernias is becoming more common. Many reviewers recommend the routine use of computer tomography to evaluate patients who present with likely lumbar hernia, particularly in the setting of blunt trauma where simultaneous organ injury may have occurred. The sensitivity for CT scan 


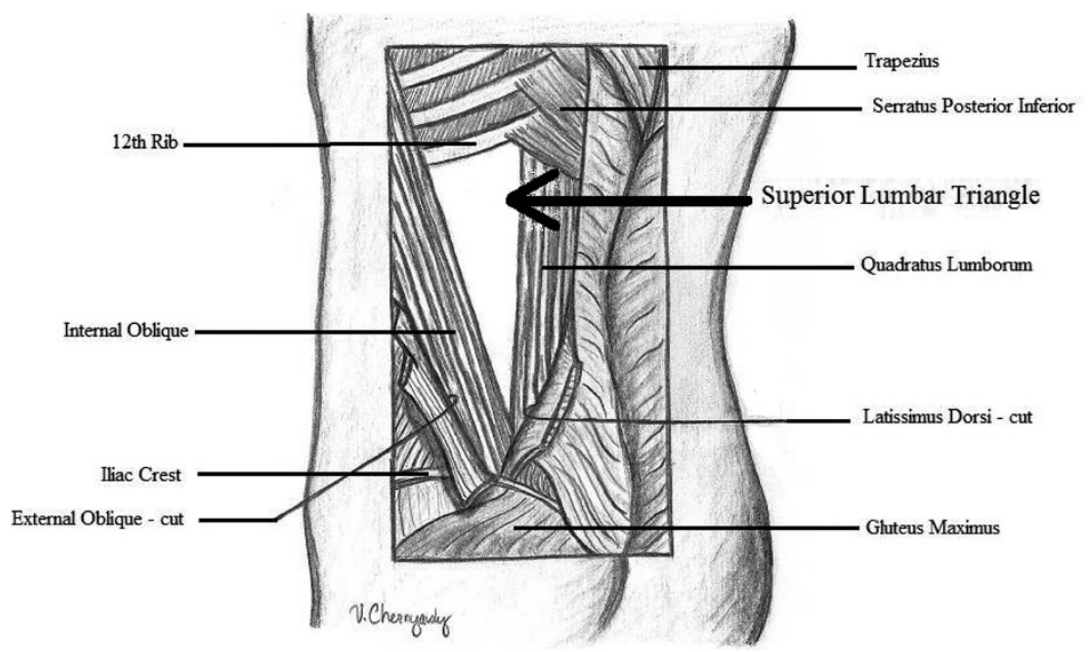

Figure 1. Superior lumbar triangle

is approximately $98 \%$ according to some reviews. Some reviewers have also found MRI useful in confirming lumbar hernia defects [8].

Lumbar hernias secondary to trauma are exceedingly rare, and different surgical repair techniques have been described. Most reports describe the use of mesh to repair the defect, which was first described by Thorek in 1950 using tantalum [9] and subsequently by Hafner in 1963 using Marlex [10]. We report the use of a biosynthetic mesh as an adjunct to repairing the defect which has the propensity to have a higher likelihood of contamination or infection secondary to traumatic etiology.

\section{Materials and Methods}

A thirty-three year-old male was involved in a motor vehicle collision (MVC) for which he underwent a midline exploratory laparotomy with repair of an avulsion of a superior mesenteric artery branch, repair of a small bowel mesenteric injury, and a sigmoid colon serosal injury. Two years later he presented with persistent dull left flank pain and a bulge, more prominent on valsalva. Patient denied any obstructive symptoms. Physical examination revealed a large, reducible lumbar hernia. Abdominal and pelvic CT scans demonstrated a $6 \times 8 \mathrm{~cm}$ lumbar hernia (Fig. 3).

Patient underwent an elective open herniorrhaphy. An oblique incision overlying the hernia was carried down to the level of the external oblique. Inspection of the inferior lumbar triangle revealed left colon in a hernia sac within an $8 \mathrm{~cm}$ hernia defect. The superior lumbar triangle was intact. A $6 \mathrm{x}$ $6 \mathrm{~cm}$ piece of AlloDerm (LifeCell Corporation, Branchburg, NJ) was used to repair the defect. It was placed as an interposition graft and sutured supero-medially to the aponeurotic



Figure 2. Inferior lumbar triangle 




Figure 3. CT scan demonstrating left lumbar hernia

fibers of the internal oblique, inferiorly to the periosteum of the iliac crest, and laterally to the quadratus lumborum (Fig. 4). A Jackson-Pratt drain was placed over the internal oblique and the external oblique was reapproximated laterally to the level of the paraspinous muscle.

\section{Results}

The patient tolerated the procedure well, returning home on post-operative day one. He experienced an unremarkable post-operative course without any complications. The drain was removed two weeks after the operation. The patient had no evidence of recurrence at six months follow-up.

\section{Discussion}

The low prevalence of lumbar hernias and the challenges involved in their repair has led to a multitude of different surgical approaches. The first described, and since modified, primary repair was described by Dowd in 1907. His repair was done using a flap from the aponeurosis of the gluteus maximus muscle, rotated and sutured to the lumbar fascia, the external oblique, and the latissimus dorsi muscles [11]. Primary closure has a high recurrence rate due to the limited fascial strength and high tension of the repair. This is also in part due to the anatomic limitations of finding an appropriate landmark to anchor the sutures and significant attenuation of the adjacent tissue, specifically the internal and external obliques.

More current surgical techniques have employed prosthetic polypropylene mesh, Marlex or Prolene, to bridge the defect. The Dowd-Ponka technique involves making an incision over the hernia site, reducing the sac, and placement of a prosthetic mesh which is sutured to the external oblique, latissimus dorsi, and the lumbar periosteum [12]. Closure is completed by approximating of the external oblique and latissimus dorsi followed by constructing of a gluteal fascia flap to cover the defect.

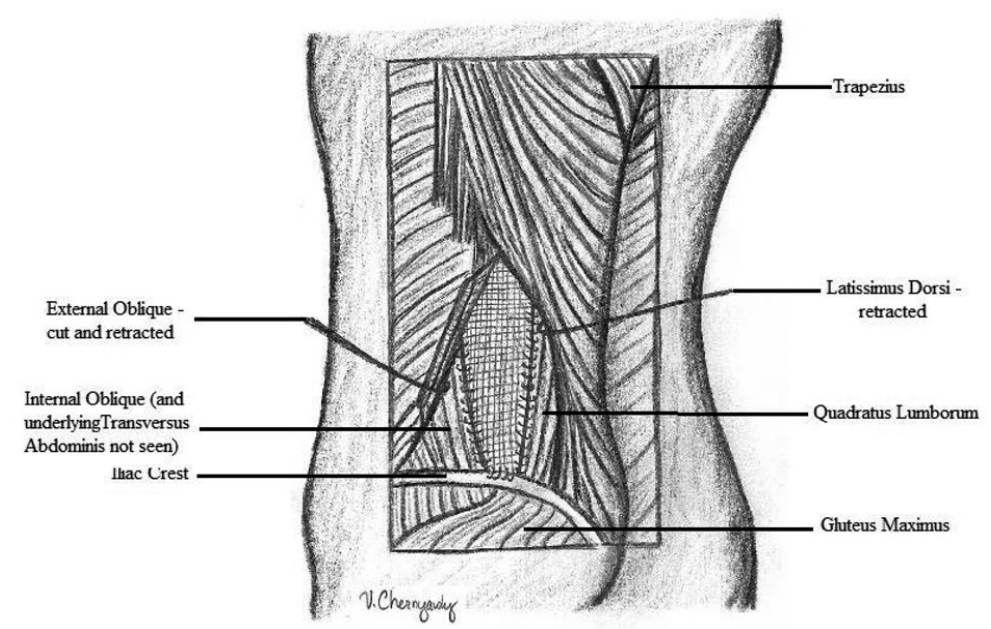

Figure 4. Repair of traumatic lumbar hernia with biologic mesh 
Both laparoscopic and retroperitoneoscopic repair using prosthetic mesh has also been described [13-16]. Arca et al. reviewed 7 laparoscopic lumbar hernia repairs, 5 of which were traumatic, performed at their institutions. These repairs involved the use of polypropylene or polytetrafluoroethylene (PTFE) mesh which was cut to overlap the fascial edges of the hernia at least $4 \mathrm{~cm}$ in all directions. In superior lumbar hernias repairs, mesh was secured to the 12 th rib.

For inferior lumbar hernia repairs, mesh was secured to the iliac crest. This involved the use of an orthopedic drill bit to create holes in the iliac crest for suturing and anchoring of the mesh. Short term follow up for this group were without recurrence [16].

The specific issue of traumatic lumbar hernia has also been in addressed in the literature. Burt et al reviewed 66 cases of lumbar hernias secondary to blunt trauma [4]. They reported $70 \%$ of traumatic hernias occurred in the inferior lumbar triangle, $71 \%$ were associated with a motor vehicle collision, and $70 \%$ of these patients were restrained in the vehicle. $61 \%$ had associated intra-abdominal injuries. Burt et al also reported that contents of the lumbar hernia have included fat (42\%), colon (41\%), and small bowel (32\%). On initial presentation 5\% were incarcerated. Timing and type of repair had been affected by other associated injuries such as bowel perforation or delay in diagnosis [17].

All existing reports of lumbar hernia repair have involved the use of synthetic mesh. Synthetic mesh for hernia repair has been associated with complications including infection, bowel obstruction, and fistula formation [18]. Using a synthetic mesh in a contaminated wound is contraindicated secondary to high rates of infection and recurrence. More recently, the use of human acellular dermis (Alloderm: LifeCell Corporation, Branchburg NJ) has allowed for a single stage operation for ventral hernia repair in the setting of contamination. This bioprosthesis acts as a scaffold for tissue regeneration, revascularization, and repopulation of cells after implantation. Overtime the material is entirely degraded and is less prone to developing adhesions. The downside to this mesh is cost and several reports in the literature of higher recurrence rates. Traumatic lumbar hernias have associated intra-abdominal injuries and repair may be needed in a potentially contaminated or infected field. Delayed repairs have been reported however, may lead to risks of incarceration or strangulation [19]. A biosynthetic mesh may be an appropriate modality in such instances.

In summary, lumbar hernias are a rare occurrence. There are reports in the literature using laparoscopic, open, or a combination of these techniques for surgical repair. All reports have described using a synthetic mesh for the repair. There are no reports on the use of biologically inert material for repair of traumatic lumbar hernias. The potential advantage of this material in the setting of a traumatic lumbar hernia should be considered. It may be ideal for immediate repair of a symptomatic or incarcerated traumatic lumbar hernia if there is gross contamination or exposed bowel.

\section{References}

1. Moreno-Egea A, Baena EG, Calle MC, Martinez JA, Albasini JL. Controversies in the current management of lumbar hernias. Arch Surg. 2007;142(1):82-88.

2. Orcutt TW. Hernia of the superior lumbar triangle. Ann Surg. 1971;173(2):294-297.

3. Loukas M, Tubbs RS, Shoja M. Lumbar hernia, anatomical basis and clinical aspects. Surg Radiol Anat. 2008;30(7):609-610.

4. Burt BM, Afifi HY, Wantz GE, Barie PS. Traumatic lumbar hernia: report of cases and comprehensive review of the literature. J Trauma. 2004;57(6):1361-1370.

5. Salemis NS, Nisotakis K, Gourgiotis S, Tsohataridis E. Segmental liver incarceration through a recurrent incisional lumbar hernia. Hepatobiliary Pancreat Dis Int. 2007;6(4):442-444.

6. Steerman SN, Steerman PH. Scoliotic lumbar hernia as a cause of colonic obstruction. J Am Coll Surg. 2004;199(1):162.

7. Stamatiou D, Skandalakis JE, Skandalakis LJ, Mirilas P. Lumbar hernia: surgical anatomy, embryology, and technique of repair. Am Surg. 2009;75(3):202-207.

8. Armstrong O, Hamel A, Grignon B, JM ND, Hamel O, Robert R, Rogez JM. Lumbar hernia: anatomical basis and clinical aspects. Surg Radiol Anat. 2008;30(7):533537; discussion 609-510.

9. Candage R, Jones K, Luchette FA, Sinacore JM, Vandevender D, Reed RL, 2nd. Use of human acellular dermal matrix for hernia repair: friend or foe? Surgery. 2008;144(4):703-709; discussion 709-711.

10. Hafner CD, Wylie JH, Jr., Brush BE. Petit's lumbar hernia: repair with Marlex mesh. Arch Surg. 1963;86:180186.

11. Swartz WT. Lumbar Hernia. Hernia, 2nd ed.1978; 409426.

12. Skandalakis LJ, Skandalakis JE, Skandalakis PN. Surgical anatomy and technique. Springer, 3rd ed. 2008.

13. Habib E. Retroperitoneoscopic tension-free repair of lumbar hernia. Hernia. 2003;7(3):150-152.

14. Thorek M. Lumbar Hernia. J Int Coll Surg. 1950;14(4):367-393.

15. Madan AK, Ternovits CA, Speck KE, Pritchard FE, Tichansky DS. Laparoscopic lumbar hernia repair. Am Surg. 2006;72(4):318-321.

16. Arca MJ, Heniford BT, Pokorny R, Wilson MA, Mayes J, Gagner M. Laparoscopic repair of lumbar hernias. J Am Coll Surg. 1998;187(2):147-152.

17. Esposito TJ, Fedorak I. Traumatic lumbar hernia: case report and literature review. J Trauma. 1994;37(1):123-126.

18. Iannitti DA, Hope WW, Norton HJ, Lincourt AE, Mil- 
likan K, Fenoglio ME, Moskowitz M. Technique and outcomes of abdominal incisional hernia repair using a synthetic composite mesh: a report of 455 cases. J Am Coll Surg. 2008;206(1):83-88.
19. Bathla L, Davies E, Fitzgibbons RJ, Jr., Cemaj S. Timing of traumatic lumbar hernia repair: is delayed repair safe? Report of two cases and review of the literature. Hernia. 2011;15(2):205-209. 